\title{
Theory of alkali-metal-induced reconstructions of fcc(100) surfaces
}

\author{
Christensen, Ole Bøssing; Jacobsen, Karsten Wedel
}

Published in:

Physical Review B

Link to article, DOI:

10.1103/PhysRevB.45.6893

Publication date:

1992

Document Version

Publisher's PDF, also known as Version of record

Link back to DTU Orbit

Citation (APA):

Christensen, O. B., \& Jacobsen, K. W. (1992). Theory of alkali-metal-induced reconstructions of fcc(100) surfaces. Physical Review B, 45(12), 6893-6898. https://doi.org/10.1103/PhysRevB.45.6893

\section{General rights}

Copyright and moral rights for the publications made accessible in the public portal are retained by the authors and/or other copyright owners and it is a condition of accessing publications that users recognise and abide by the legal requirements associated with these rights.

- Users may download and print one copy of any publication from the public portal for the purpose of private study or research.

- You may not further distribute the material or use it for any profit-making activity or commercial gain

- You may freely distribute the URL identifying the publication in the public portal

If you believe that this document breaches copyright please contact us providing details, and we will remove access to the work immediately and investigate your claim. 


\title{
Theory of alkali-metal-induced reconstructions of fcc(100) surfaces
}

\author{
O. B. Christensen and K. W. Jacobsen \\ Laboratory of Applied Physics, Technical University of Denmark, DK-2800 Lyngby, Denmark
}

(Received 27 June 1991)

\begin{abstract}
Calculations of missing-row reconstruction energies of the fcc(100) surfaces of the metals $\mathrm{Al}, \mathrm{Ni}$, $\mathrm{Pd}, \mathrm{Pt}, \mathrm{Cu}, \mathrm{Ag}$, and $\mathrm{Au}$ have been performed with the effective-medium theory with and without the presence of a potassium overlayer. It is shown that the tendency to reconstruct in the presence of adsorbed $\mathrm{K}$ is largest for Ag. This is in accordance with recent experiments indicating a potassiuminduced missing-row reconstruction for $\mathrm{Ag}$, but not for other metals. The tendency is shown to be related to the relatively low bulk modulus of silver. Differences from the well-known alkali-metalinduced reconstruction of $\mathrm{fcc}(110)$ surfaces are pointed out.
\end{abstract}

\section{INTRODUCTION}

Alkali metals are known to induce missing-row reconstructions on the (110) surfaces of most fcc metals at low coverages ${ }^{1}$ and the nature of this reconstruction has received considerable theoretical interest. ${ }^{2-4}$ Theoretical studies of the clean $\mathrm{fcc}(110)$ surfaces show ${ }^{3,5}$ that the metals which do not exhibit missing-row reconstructions for the clean surfaces are only slightly stable with reconstruction energies of the order of $10 \mathrm{meV}$ per atom. Alkali-metal atoms chemisorbed on a missing-row reconstructed surface are positioned in the "valleys" formed by the missing rows, and the higher effective coordination number of the alkali-metal atoms in these valleys leads to an additional binding of the order of $0.1 \mathrm{eV}$ compared to chemisorption on an unreconstructed surface. ${ }^{3}$ A small coverage of alkali atoms can therefore induce a missing-row reconstruction of the (110) surfaces.

Recently, a potassium-induced $(1 \times 2)$ missing-row reconstruction of the more closely packed (100) surface of $\mathrm{Ag}$ was reported. ${ }^{6,7}$ No similar reconstruction has been observed for $\mathrm{Al}, \mathrm{Ni}, \mathrm{Pd}, \mathrm{Cu}$, and $\mathrm{Ir} .{ }^{6}$ In order to understand the nature of this reconstruction and to give an indication of whether or not the (100) surfaces of other fcc metals can be expected to exhibit similar reconstructions, we have performed a series of effective-medium reconstruction-energy calculations for aluminum, late transition metals, and noble metals. In the following we shall present results which show that silver is indeed the most likely candidate for this kind of reconstruction among the metals $\mathrm{Al}, \mathrm{Ni}, \mathrm{Pd}, \mathrm{Pt}, \mathrm{Cu}, \mathrm{Ag}$, and $\mathrm{Au}$, and we shall relate this to the relatively small bulk modulus of silver.

\section{EFFECTIVE-MEDIUM THEORY}

The basic idea behind the effective-medium theory ${ }^{8}$ is to estimate the binding energy of an atom in a metallic system by a comparison to a simpler reference system (the "effective medium"). The reference system is usually chosen as the perfect fcc crystal with an appropriate lattice constant or as the atom embedded in a homogeneous electron gas of a suitable density. The effective-medium theor $\mathrm{y}^{8}$ is derived from density-functional theory and utilizes the stationary property of the energy functional with respect to variations in the electron density. The electron density for a system of atoms is approximated by a superposition of screened-atom densities calculated by embedding each of the atoms in a homogeneous electron gas. This ansatz for the density leads to an expression ${ }^{8}$ for the binding energy of the system which can be written

$$
E_{\mathrm{tot}}=\sum_{\text {atoms } i} E_{c, Z_{i}}\left(\bar{n}_{i}\right)+\sum_{\text {atoms } i} E_{\mathrm{AS}}^{(i)}+E_{1-\mathrm{el}},
$$

where the sums run over all the atoms in the system. The first term in Eq. (1) contains the cohesive functions $E_{c, Z_{i}}$ which are functions of the embedding densities $\bar{n}_{i}$. The embedding density of a given atom is the average electron density in which the atom is situated and is evaluated as a sum of contributions from the other atoms in the system. The cohesive functions depend only on the atomic number $Z_{i}$ of the atom and describe how the energy changes with volume locally around an atom. The cohesive functions for the metals discussed here all exhibit a single minimum at a density $n_{0, Z_{i}}$ characteristic of the metal. This density determines the equilibrium lattice constant of the metal and the second derivative of the cohesive function around the minimum is proportional to the bulk modulus of the metal. ${ }^{8}$ The atomicsphere correction energy $E_{\mathrm{AS}}$ describes the difference in electrostatic interaction between the atoms in the system under consideration and in a close-packed (fcc) arrangement (where the electrostatic interactions are already included in the cohesive function). Finally, the one-electron energy $E_{1-e l}$ describes the energy difference due to the difference in the local density of states for an atom in the metallic system compared with the same atom in the reference system. This term can be neglected in most situations for simple metals and late transition metals, ${ }^{8}$ but has been shown recently to give important contributions if the atoms form strong covalent bonds as is the case in surface diffusion exchange processes. ${ }^{9}$ In the present work the one-electron energy is neglected. Details of the calculation procedure can be found in Ref. 8 . 


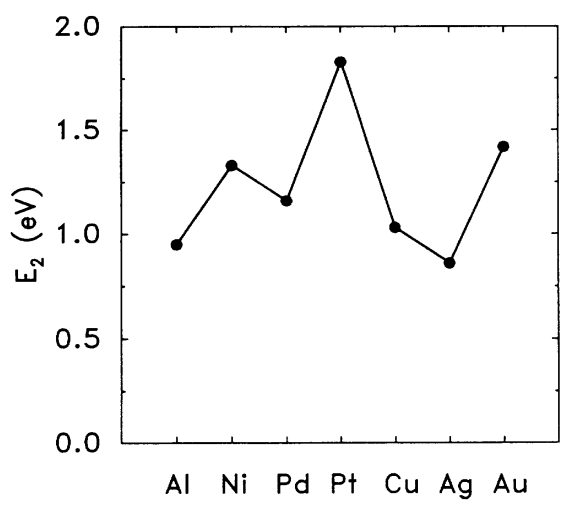

FIG. 1. The effective-medium parameter $E_{2}$ for the metals investigated. This parameter is roughly proportional to the bulk modulus of the metal.

The effective-medium theory has been applied in a number of studies of energetics of metal surfaces including descriptions of clean surface relaxations and reconstructions, $, 8,3$ adsorbate-induced relaxations ${ }^{10}$ and reconstructions, ${ }^{3,11}$ self-diffusion at surfaces, ${ }^{9}$ surface premelting, ${ }^{12}$ and small metal clusters. ${ }^{13}$

Before we continue to analyze the missing-row reconstructions we shall discuss some key concepts of the effective-medium theory. This requires a short presentation of some of the parameters in the theory, their interpretation, and their relation to quantities which can be determined experimentally. The cohesive energy of a given metal is simply the value $E_{0}$ of the cohesive function at the minimum point (i.e., at the density $n_{0}$ ). The curvature of the cohesive function at the minimum is usually described by a parameter $E_{2}=1 / 2 n_{0}^{2} d^{2} E_{c} / d \bar{n}^{2}$, and the bulk modulus of the metal is roughly proportional to this parameter. (The ratio between the two varies with

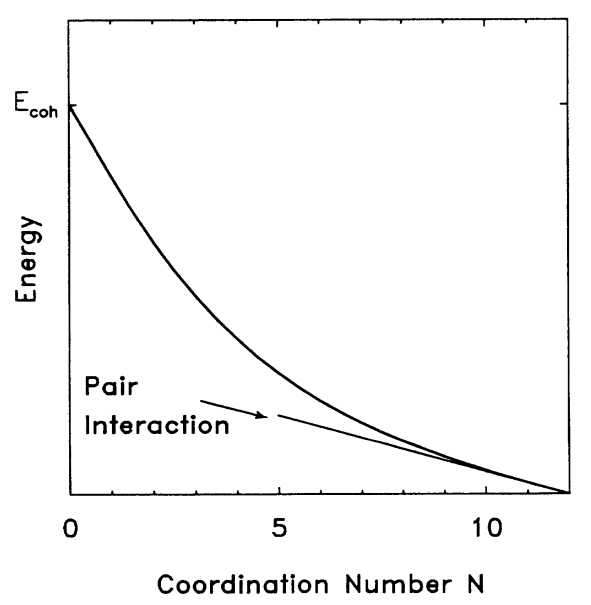

FIG. 2. Energy of an atom as a function of the coordination number in an effective nearest-neighbor model (schematic). The effective pair interaction evaluated in the bulk configuration $(N=12)$ is indicated by the straight line. It can be seen that this contribution is not directly related to the cohesive energy $E_{\text {coh }}$ of the crystal.

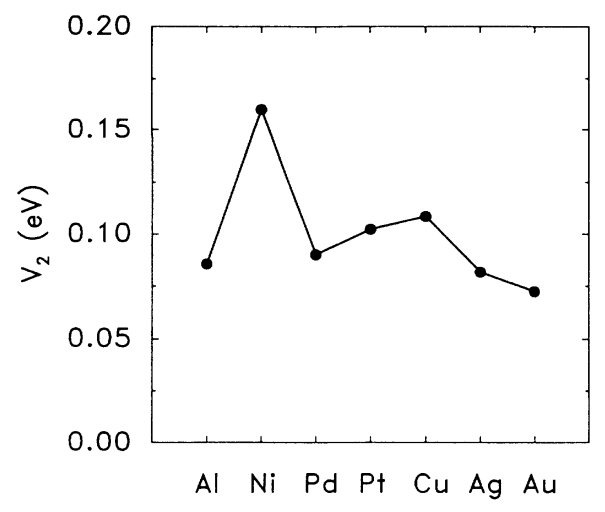

FIG. 3. The pair interaction strength $V_{2}$ of Eq. (2).

about $20 \%$ for the metals studied here.) The curvature $E_{2}$ is to a large extent determined by the kinetic energy of the electrons. The values of $E_{2}$ for the metals considered in the present work are shown in Fig. 1. The strength of the atomic-sphere correction energy is given by an electrostatic parameter $V_{2}$ which is also the strength of the effective pair potential between the atoms in the metal. ${ }^{8,14}$ The effective pair potential $V_{2}$ enters in an expansion of the energy $E\left(N_{1}, N_{2}\right)$ of an atom as a function of nearestneighbor $\left(N_{1}\right)$ and next-nearest-neighbor $\left(N_{2}\right)$ coordination numbers. ${ }^{14,15}$ The expansion is done around the bulk equilibrium configuration with 12 nearest neighbors and 6 next-nearest neighbors. All interatomic distances are fixed at their bulk equilibrium values:

$$
\begin{aligned}
E\left(N_{1}, N_{2}\right) \simeq & V_{2}\left(12-N_{1}\right)+\frac{1}{2} V_{3}\left(12-N_{1}\right)^{2} \\
& +V_{2}^{\prime}\left(6-N_{2}\right) .
\end{aligned}
$$

In Fig. 2 we sketch the energy of an atom as a function of coordination number in an effective nearest-neighbor model. The energy difference between the situations with $N_{1}=12$ and 0 is, of course, the cohesive energy. The ef-

TABLE I. The effective nearest- and nextnearest-neighbor pair interaction $V_{2}$ and $V_{2}^{\prime}$, and the effective nearest-neighbor three-body interaction $V_{3}$ from Eq. (2). The interaction energies have been calculated from the usual effective-medium parameters using the formulas in Refs. 3 and 14. The effective-medium parameters have been evaluated in the local-density approximation for an atom embedded in a homogeneous electron gas (Ref. 8) with an additional adjustment of the parameters $s_{0}, E_{2}$, and $\eta_{1}$ (see Refs. 3 and 14) as to reproduce the experimental lattice constants, bulk moduli, and shear moduli $\left(C_{44}\right)$. The experimental values have been taken from Ref. 23. All quantities in the table are in units of $\mathrm{eV}$.

\begin{tabular}{cccc}
\hline \hline Metal & $V_{2}$ & $V_{2}^{\prime}$ & $V_{3}$ \\
\hline $\mathrm{Al}$ & 0.073 & 0.0204 & 0.0040 \\
$\mathrm{Ni}$ & 0.160 & 0.0356 & 0.0007 \\
$\mathrm{Pd}$ & 0.090 & 0.0136 & 0.0064 \\
$\mathrm{Pt}$ & 0.102 & 0.0157 & 0.0140 \\
$\mathrm{Cu}$ & 0.109 & 0.0246 & 0.0023 \\
$\mathrm{Ag}$ & 0.082 & 0.0146 & 0.0034 \\
$\mathrm{Au}$ & 0.072 & 0.0131 & 0.0116 \\
\hline \hline
\end{tabular}




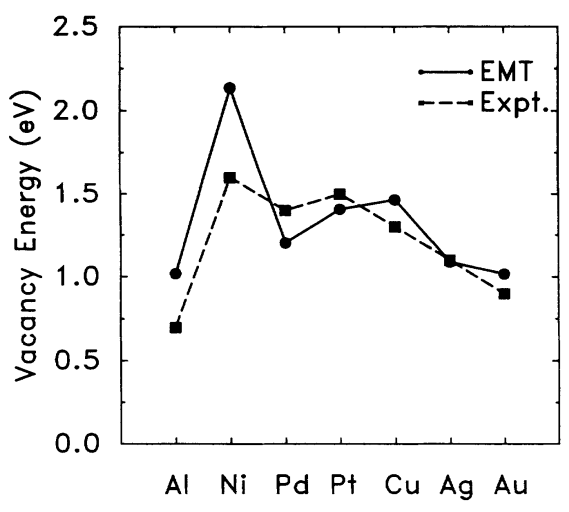

FIG. 4. Vacancy formation energy for the seven metals. The effective-medium estimate (circles/solid line) from Eq. (2) is proportional to $V_{2}$ (Fig. 3). The trend in the experimental results (Ref. 16) (squares/dashed line) is reproduced.

fective pair potential is the slope of the curve at $N_{1}=12$ as indicated by the straight line in the figure. It is seen that the effective pair potential is not determined by the cohesive energy of the metal. In fact the strength of the effective pair potential $V_{2}$ is proportional to the shear modulus ${ }^{8,14} C_{44}$. Its value for the metals studied here are shown in Fig. 3 and in Table I. The next-nearestneighbor pair potential $V_{2}^{\prime}$ has exactly the same trend as $V_{2}$ and is about a factor of 4 smaller, as can be seen in Table I. Since the nearest-neighbor pair potential dominates the variation of energy with the coordination number around the bulk value, the same trend should be found in the vacancy formation energy. This is indeed the case as it may be seen in Fig. 4.

The three-body potential $V_{3}$ in Eq. (2), which comes from the curvature of the energy as a function of coordination number (Fig. 2), is an order of magnitude smaller than the pair potential and is a linear combination of $E_{2}$ and $V_{2}$ where the coefficient of $E_{2}$ is positive and the coefficient of $V_{2}$ is negative. ${ }^{14}$ Metals with a large bulk modulus relative to the shear modulus $C_{44}$ therefore have relatively large positive values of the three-body interactions. The three-body potential has a completely differ-

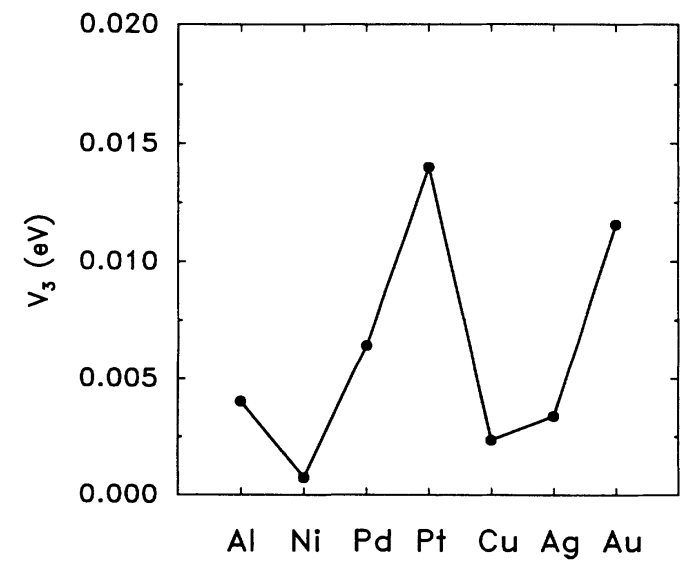

FIG. 5. Three-body interaction strength $V_{3}$ of Eq. (2). ent trend than the pair potential as can be seen from Fig. 5.

\section{RESULTS AND DISCUSSION}

We now turn to a comparison of the $(1 \times 2)$ missing-row reconstructions of clean (110) and (100) fcc metal surfaces based on the expansion in Eq. (2). In this model the relaxations of the atomic positions are neglected. The reconstruction energies can therefore be evaluated from the number of nearest and next-nearest neighbors of surface atoms in the reconstructed and the unreconstructed surface structures. The $(1 \times 2)$ reconstruction of the (110) surface does not involve any net breaking of nearest-neighbor bonds and the reconstruction energy therefore does not depend on the pair potential $V_{2}$. The reconstruction energy per $(1 \times 1)$ unit cell $\Delta E^{(110)}$ is given by ${ }^{14}$

$$
\Delta E^{(110)}=-2 V_{3}+V_{2}^{\prime}
$$

and is shown in Fig. 6. The trend is the same (with opposite sign) as in the three-body potential (Fig. 5) and the metals $\mathrm{Pt}$ and $\mathrm{Au}$, which are known experimentally to reconstruct, indeed have negative reconstruction energies. This can be traced back to the fact that these metals have particularly large bulk moduli compared to their shear moduli, due to the extended core and $d$ states.

The suggested $(1 \times 2)$ missing-row reconstruction of the $\operatorname{Ag}(100)$ surface has a character completely different from the (110) missing-row reconstruction. The reconstruction of the clean surface involves breaking nearest-neighbor bonds and the reconstruction energy $\Delta E^{(100)}$ per $1 \times 1$ unit cell therefore depends on the nearest-neighbor pair potential:

$$
\Delta E^{(100)}=V_{2}+2 V_{2}^{\prime}+3 V_{3} .
$$

The trend in the reconstruction energy is determined by the pair potential as can be seen from a comparison of

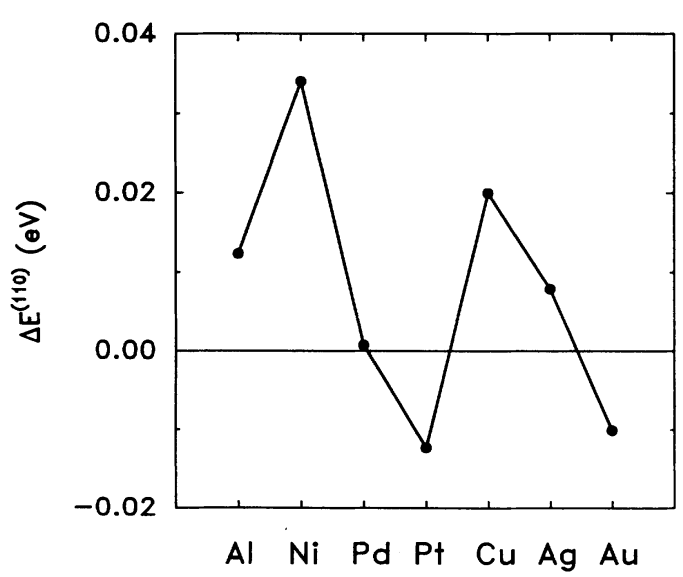

FIG. 6. Missing-row reconstruction energy of the (110) surface per unreconstructed unit cell. In this reconstruction the total number of nearest-neighbor bonds is not changed, and the reconstruction energy is correlated to the three-body term $V_{3}$ (Fig. 5). 


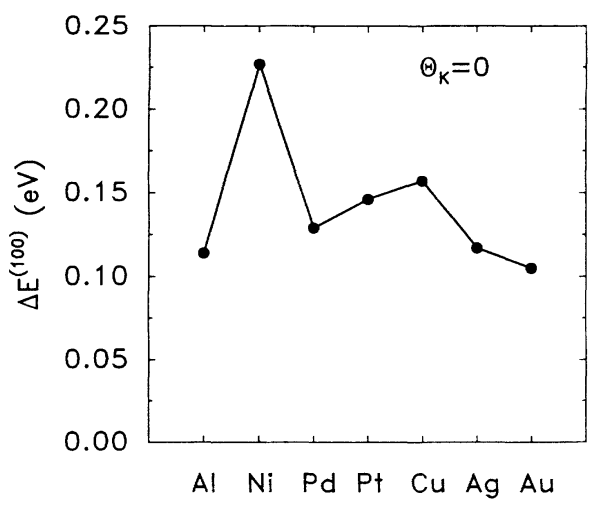

FIG. 7. Missing-row reconstruction energy of the (100) surface per unreconstructed unit cell at zero $\mathrm{K}$ coverage. The dominating effect is the breaking of nearest-neighbor bonds in the reconstruction. This is reflected in the fact that the observed trend is the same as the trend in the pair interaction strength $V_{2}$ (Fig. 3).

Figs. 7 and 3. The absolute numbers are an order of magnitude higher than for the reconstruction of the (110) surface.

As already mentioned in the introduction, alkali-metal atoms bind more strongly on the missing-row reconstructed (110) surface than on the unreconstructed surface. At a rather small critical coverage this causes the reconstruction energy of the surface to change sign, and an alkali-metal-induced reconstruction takes place. The binding energy of, say, potassium is also enhanced on the $(1 \times 2)$ missing-row reconstructed $(100)$ surface compared with the unreconstructed one, but for this surface the cost in the reconstruction of the clean surface is much higher and the final result is a delicate balance between these two contributions. If we naively assume that the difference between the chemisorption energy of potassium on an unreconstructed and on a reconstructed (100) surface is independent of the the kind of substrate metal, we would expect the reconstruction energy for the potassium-covered surface to show the same trend as for the clean metal surface (Fig. 7). However, as we shall now see, this is not the case.

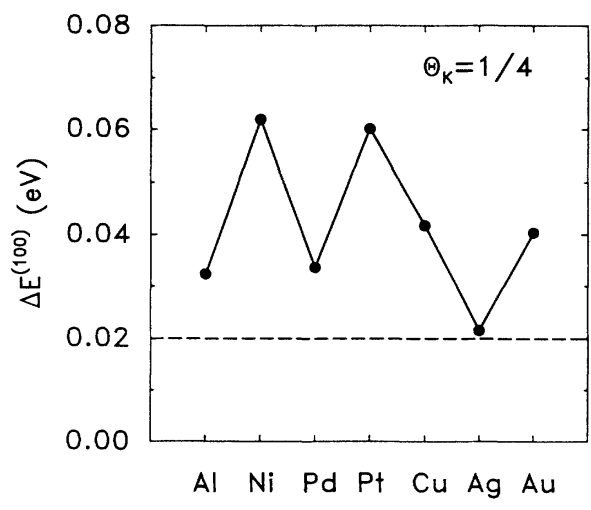

FIG. 8. Effective-medium missing-row reconstruction energy of the (100) surface per unreconstructed unit cell at a potassium coverage of $\frac{1}{4} \mathrm{~K}$ atoms per surface atom in the unreconstructed surface structure. The observed trend is roughly the same as the trend in the bulk-modulus parameter $E_{2}$ of Fig. 1 . The dashed line at $20 \mathrm{meV}$ indicates an estimated shift of the energy zero due to the dipole interaction energies. Ag is seen to be only marginally stable.

We have performed a series of effective-medium calculations for the $(1 \times 2)$ missing-row reconstruction energies of the $(100)$ surfaces with an ordered $c(2 \times 2)$ potassium overlayer of coverage $\frac{1}{4}$. All atoms down to the second full layer of substrate atoms (9 and 11 atoms, respectively) were allowed to relax to their equilibrium positions. The resulting reconstruction energies are shown in Fig. 8 and in Table II. Due to the enhanced binding of the potassium on the missing-row reconstructed surfaces the reconstruction energies are an order of magnitude smaller than those found for the clean surfaces, and the trend is completely different. The trend in the reconstruction energies is therefore not determined by the effective substrate-substrate pair interaction.

As can be seen from a comparison of Figs. 8 and 1, the reconstruction energy roughly follows the trend in $E_{2}$, the curvature of the cohesive function for the substrate metal. The reason for this behavior is the following: A chemisorbed potassium atom positions itself outside a metal surface with bond distances which en-

TABLE II. Reconstruction energies for the (100) surfaces in eV per unreconstructed surface unit cell. The table contains calculated reconstruction energies for the clean $(\theta=0)$ surfaces with $\left(\Delta E_{\mathrm{rel}}\right)$ and without $\left(\Delta E_{\mathrm{unrel}}\right)$ relaxations of the atomic positions. For the potassium-covered $\left(\theta=\frac{1}{4}\right)$ surface the result of a fully relaxed calculation $\left(\Delta E_{\mathrm{rel}}^{\mathrm{EMT}}\right)$ is shown. The dipole energy difference $\left(\Delta E_{\text {dip }}\right)$ at $\theta=\frac{1}{4}$ is estimated from the Topping model for $\mathrm{Al}, \mathrm{Ni}, \mathrm{Pt}$, and $\mathrm{Ag}$ (see text). For $\mathrm{Pd}, \mathrm{Cu}$, and $\mathrm{Au}$ we have set the dipole energy equal to the result for $\mathrm{Ag}$. The last column shows the total estimated reconstruction energy at a potassium coverage of $\frac{1}{4}$.

\begin{tabular}{cccccc}
\hline \hline Metal & $\Delta E_{\text {unrel }}(\theta=0)$ & $\Delta E_{\text {rel }}(\theta=0)$ & $\Delta E_{\text {rel }}^{\text {EMT }}\left(\theta=\frac{1}{4}\right)$ & $\Delta E_{\text {dip }}$ & $\Delta E_{\text {rel }}^{\text {tot }}\left(\theta=\frac{1}{4}\right)$ \\
\hline $\mathrm{Al}$ & 0.126 & 0.114 & 0.032 & -0.011 & 0.020 \\
$\mathrm{Ni}$ & 0.233 & 0.227 & 0.062 & -0.015 & 0.047 \\
$\mathrm{Pd}$ & 0.136 & 0.129 & 0.034 & $(-0.020)$ & 0.014 \\
$\mathrm{Pt}$ & 0.175 & 0.146 & 0.060 & -0.019 & 0.041 \\
$\mathrm{Cu}$ & 0.165 & 0.157 & 0.042 & $(-0.020)$ & 0.022 \\
$\mathrm{Ag}$ & 0.121 & 0.117 & 0.022 & -0.020 & 0.002 \\
$\mathrm{Au}$ & 0.133 & 0.105 & 0.040 & $(-0.020)$ & 0.020 \\
\hline \hline
\end{tabular}


sure that its embedding density is close to the optimal one which gives the minimum value of the cohesive function for potassium. This is a consequence of the much quicker variation of the potassium embedding density $\bar{n}$ with adsorbate-substrate distance than the variation of the same quantity for the surface atoms. An adsorbed potassium atom interacts strongly with the surface atoms and is able to screen out the electrostatic interactions between substrate surface atoms, which gave rise to the rather large reconstruction energies for the clean (100) surfaces. The potassium atom also gives a contribution to the embedding densities of the surface metal atoms. The embedding density of a surface atom on the clean reconstructed and unreconstructed surfaces are different due to the different coordination numbers of the surface atom. This means that the additional contribution to the embedding densities of the surface atoms from the potassium atom will give rise to different energy changes on the two surfaces because of the nonlinearity of the cohesive function. The magnitude of this difference is proportional to the curvature $E_{2}$ of the cohesive function and is therefore related to the bulk modulus; in consequence, the particularly small value of the reconstruction energy for silver can be understood from the low bulk modulus of this metal. Note that the effect comes from a difference in the substrate-potassium interaction due to a difference in the coordination number of the substrate surface atoms. It is therefore not described in a model with only pairwise interactions.

It is seen in Table II that all the calculated reconstruction energies are positive. This is due to the fact that the effective-medium calculation is based on spherical charge densities and therefore does not include the electrostatic dipole-dipole interaction between the potassium atoms. The effective dipole from a potassium atom is smaller when the atom is chemisorbed on a reconstructed surface than on the unreconstructed one because of the more efficient screening of an atom positioned in one of the missing-row valleys. The dipole energy is therefore lower at the reconstructed surface.

We have performed a rough estimate of the dipole energy difference. The reduction of adsorbate dipole moment with increasing coverage has been estimated in the Topping model. ${ }^{17}$ We have made the simple assumption of a charge transfer distributed evenly on all surface atoms close to the adsorbate. In other words, we assume the small-coverage dipole moment to be proportional to the height of the alkali-metal atom over a surface level, which is determined by averaging over the surface atoms close to the alkali-metal atom. All heights are obtained from EMT calculations. The two parameters required by the Topping model are obtained by fitting to experimental work-function data where available $\left[\mathrm{Al}(111),{ }^{18}\right.$ $\mathrm{Ni}(100),{ }^{19} \mathrm{Ni}(111),{ }^{20} \mathrm{Ag}(100),{ }^{7}$ and $\mathrm{Pt}(111)$ (Ref. 21)]. The estimated values for the change in the dipole energy when the reconstruction takes place is shown in Table II. ${ }^{22}$ For the transition metals and noble metals the dipole energy leads to a lowering of the reconstruction energy by approximately $20 \mathrm{meV}$ as indicated by the dashed line in Fig. 8. The inclusion of the dipole energy makes the potassium covered $\mathrm{Ag}(100)$ surface only marginally stable by a few meV toward the missing-row reconstruction while the unreconstructed surfaces of the other metals still remain stable.

The model we have used is clearly not able to quantitatively predict the reconstruction energies with an accuracy of a few meV. However, the effective-medium calculations indicate a clear trend in the reconstruction energies where metals with low bulk moduli show the strongest tendency to reconstruct. The estimates of the dipole energies from the Topping model show that the sign of the reconstruction energy for the potassiumcovered $\mathrm{Ag}(100)$ surface may easily be negative when the dipole energy is included. On the other hand, the dipole energies are seen to be so similar for the different substrate metals that we believe the calculated trend in the reconstruction energies will not be changed by the dipole contribution.

\section{CONCLUSION}

The effective-medium calculations for $\mathrm{Al}, \mathrm{Ni}, \mathrm{Pd}, \mathrm{Pt}$, $\mathrm{Cu}, \mathrm{Ag}$, and $\mathrm{Au}$ indicate that the (100) surfaces of metals with low bulk moduli have a tendency to exhibit a missing-row reconstruction when covered with $\frac{1}{4}$ monolayer potassium. This is in agreement with the observation $^{6,7}$ of such a reconstruction of $\operatorname{Ag}(100)$. The trend in the reconstruction energies is completely different from the one observed for the $(1 \times 2)$ missing-row reconstruction of the clean (110) surfaces where metals with a large bulk modulus compared with the shear modulus reconstruct.

\section{ACKNOWLEDGMENTS}

Many stimulating discussions with J. K. Nørskov are gratefully appreciated. The research has been supported by the Danish Research Councils through the Center for Surface Reactivity.
${ }^{1}$ B. E. Hayden, K. C. Prince, P. J. Davies, G. Paolucci, and A. M. Bradshaw, Solid State Commun. 48, 325 (1983). C. J. Barnes, M. Q. Ding, M. Lindroos, R. D. Diehl, and D. King, Surf. Sci. 162, 59 (1983); S. M. Francis and N. V. Richardson, ibid. 152/153, 63 (1985); R. J. Behm, G. Ertl, D. K. Flynn, K. D. Jamison, and D. A. Thiel, Phys. Rev. B 36, 9267 (1987); J. W. M. Frenken,
R. L. Krans, J. F. van der Veen, E. Holub-Krappe, and K. Horn, Phys. Rev. Lett. 59, 2307 (1987).

${ }^{2}$ V. Heine and L. D. Marks, Surf. Sci. 165, 65 (1982).

${ }^{3}$ K. W. Jacobsen and J. K. Nørskov, in The Structure of Surfaces II, edited by J. F. van der Veen and M. A. Van Hove, Springer Series in Surface Sciences Vol. 11 (Springer, Berlin, 1988); K. W. Jacobsen and J. K. Nørskov, 
Phys. Rev. Lett. 60, 2496 (1988).

${ }^{4}$ K. M. Ho and K. P. Bohnen, Phys. Rev. Lett. 59, 1833

(1987); C. L. Fu and K. M. Ho, ibid. 63, 1617 (1989).

${ }^{5}$ S. M. Foiles, Surf. Sci. 191, L779 (1987).

${ }^{6}$ M. Okada, H. Tochihara, and Y. Murata, Phys. Rev. B 43, 1411 (1991).

${ }^{7}$ M. Okada, H. Tochihara, and Y. Murata, Surf. Sci. 245, 380 (1991).

${ }^{8}$ K. W. Jacobsen, J. K. Nørskov, and M. J. Puska, Phys. Rev. B 35, 7423 (1987); K. W. Jacobsen, Comments Cond. Mat. Phys. 14, 129 (1988).

${ }^{9}$ G. Kellogg and P. J. Feibelman, Phys. Rev. Lett. 64, 3143 (1990); C. Chen and T. T. Tsong, ibid. 64, 3147 (1990); L. Hansen, P. Stoltze, K. W. Jacobsen, and J. K. Nørskov, Phys. Rev. B 44, 6523 (1991).

${ }^{10}$ K. W. Jacobsen and J. K. Nørskov, Phys. Rev. Lett. 59, 2764 (1987).

${ }^{11}$ K. W. Jacobsen and J. K. Nørskov, Phys. Rev. Lett. 65, 1788 (1990); R. Feidenhans'l, F. Grey, M. Nielsen, F. Besenbacher, F. Jensen, E. Lægsgaard, I. Stensgaard, K. W. Jacobsen, J. K. Nørskov, and R. L. Johnson, ibid. 65, 2027 (1990).

${ }^{12} \mathrm{P}$. Stoltze, J. K. Nørskov, and U. Landman, Phys. Rev. Lett. 61, 440 (1988).
${ }^{13}$ O. B. Christensen, K. W. Jacobsen, J. K. Nørskov, and M. Manninen, Phys. Rev. Lett. 66, 2219 (1991); O. B. Christensen and K. W. Jacobsen (unpublished).

${ }^{14} \mathrm{~K}$. W. Jacobsen and P. Stoltze, in Ordering at Surfaces and Interfaces, edited by A. Yoshimori, T. Shinjo, and H. Watanabe (Springer, New York, 1991).

${ }^{15}$ H. Häkkinen, J. Merikoski, and M. Manninen, J. Phys. Condens. Matter 3, 2755 (1991).

${ }^{16}$ R. W. Balluffi, J. Nucl. Mater. 69\&70, 240 (1978); W. Wysick and M. Feller-Kniepmeier, ibid. 69\&70, 616 (1978).

${ }^{17}$ J. Topping, Proc. R. Soc. London Ser. A 114, 67 (1927).

${ }^{18}$ K. Horn, A. Hohlfeld, J. Somers, Th. Lindner, P. Hollins, and A. M. Bradshaw, Phys. Rev. Lett. 61, 2488 (1988).

${ }^{19}$ S. Andersson and U. Jostell, Surf. Sci. 46, 625 (1974).

${ }^{20}$ K. J. Uram, L. Ng, and J. T. Yates, Surf. Sci. 177, 253 (1986).

${ }^{21}$ M. Kiskinova, G. Pirug, and H. P. Bonzel, Surf. Sci. 133, 321 (1983).

${ }^{22}$ O. B. Christensen, Ph.D. thesis, Technical University of Denmark, 1991.

${ }^{23}$ C. Kittel, Introduction to Solid State Physics (Wiley, New York, 1971). 